\title{
Impact of Untransposed Power Lines
}

\author{
Josifs Survilo, Riga Technical University
}

\begin{abstract}
Unbalanced power lines (the conductors of which are not arranged in the vertices of equilateral triangle) without overhead grounding wire (OGW) besides Fortesque impedances $Z_{0}$ and $Z_{1}=Z_{2}$ have mutual impedances $Z_{01}, Z_{10}, Z_{02}, Z_{20}, Z_{12}, Z_{21}$. At the end of the line negative sequence and in less degree zero sequence of voltage take place having increased with the load increasing. Flat phase arrangement $110 \mathrm{kV}$ line with $X / R=4$, loaded by active load with resistance tenfold of phase conductor active resistance generates $2.54 \%$ of negative sequence and $0.79 \%$ of zero sequence. Inductive load generates less and capacitive - still more asymmetry. Stray capacitances and corona losses do not have any influence. Under asymmetric short circuits impact on relay protection depends on location of special phase: it is located laterally or in the middle of the flat arrangement. Digital relay protection can easier cope with the phase unbalance effect when the line is untransposed. Balanced voltage at the end of untransposed unbalanced power line can be obtained loading the line with unbalanced load.
\end{abstract}

Keywords - current sequence of three-phase system, flat phase arrangement, mutual impedances, self impedances, untransposed power line.

\section{INTRODUCTION}

In real power line, phase conductors rarely are situated identically with respect to each other. As a result, mutual phase inductances are slightly different for some pairs of phases. This causes unbalance of phase voltages at the end of loaded power line which is limited by standards [1]. In distribution networks European standards limit voltage unbalance factor to $2 \%$, in the USA - to $3 \%$. Requirements grow stricter with the increase of nominal voltage [2]. The voltage unbalance is undesirable since it leads to negative consequences, primarily to overheating of electric motors, generation of harmonics, additional power losses, are detrimental for power electronic converters and adjustable speed drives [1], [2]. Therefore techniques of voltage unbalance mitigation are practiced [1].

The asymmetry in ultra high voltage transmission lines [3] calculated basing on symmetrical component method brings errors. Transposition especially in high voltage power lines is expensive, while M. Gashimov et al. [4] claim that transposition cycles can not be prolonged.

In the paper, the attention is turned to analytically express parameters of untransposed power line without OGW and its effect on balanced load and on relay protection. An alternative for the line transposition is considered.

\section{EXPRESSIONS FOR IMPEDANCES}

Expressions for quantities $\boldsymbol{Q}$ (voltages $U$ and currents $I$ are meant) in phase notation $\boldsymbol{Q}_{a b c}$ and in Fortesque notation $\boldsymbol{Q}_{012}$ and their reciprocal conversions are [5]:

$\boldsymbol{Q}_{a b c}=\left[\begin{array}{c}q_{a} \\ q_{b} \\ q_{c}\end{array}\right] ; \boldsymbol{Q}_{012}=\left[\begin{array}{c}q_{0} \\ q_{1} \\ q_{2}\end{array}\right] ; \boldsymbol{Q}_{012}=\boldsymbol{A} \boldsymbol{Q}_{a b c} ; \boldsymbol{Q}_{a b c}=\boldsymbol{A}^{-1} \boldsymbol{Q}_{012}$,

where $\boldsymbol{A}$ and $\boldsymbol{A}^{-1}$ are:

$$
\boldsymbol{A}=\frac{1}{3}\left[\begin{array}{ccc}
1 & 1 & 1 \\
1 & a & a^{2} \\
1 & a^{2} & a
\end{array}\right] ; \boldsymbol{A}^{-1}=\left[\begin{array}{ccc}
1 & 1 & 1 \\
1 & a^{2} & a \\
1 & a & a^{2}
\end{array}\right] ; a=-\frac{1}{2}+j \frac{\sqrt{3}}{2}
$$

Phase conductors in flat arrangement have different distances between each other (Fig. 1). Their self impedances $z_{x x}=z_{d}$ can be considered equal, while mutual impedances $z_{x y}$ have different reactances which are: between adjacent phases $x_{s}$, between distant phases $x_{f}$. In matrix lay out branch impedances $Z_{a b c}$ are:

a

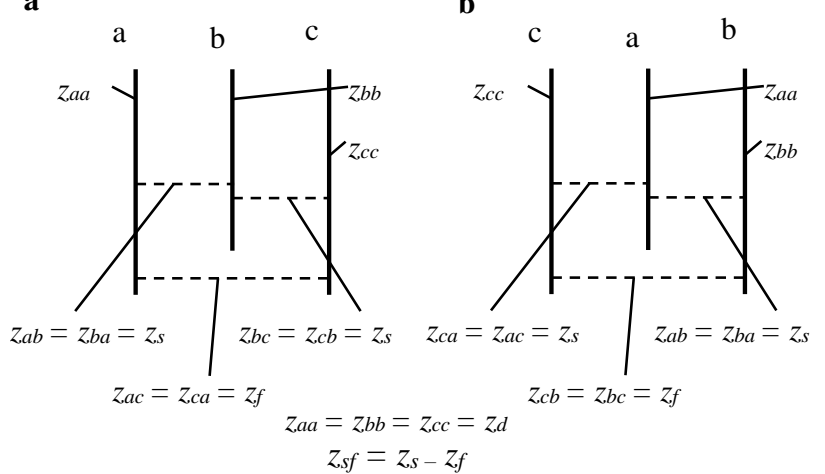

Fig. 1. Flat arrangement of phase conductors $\mathbf{a}$ - phase ' $a$ ' by side; $\mathbf{b}$ - phase ' $a$ ' in the middle.

$$
\begin{gathered}
\boldsymbol{Z}_{a b c}=\left[\begin{array}{ccc}
z_{d} & z_{a b} & z_{a c} \\
z_{b a} & z_{d} & z_{b c} \\
z_{c a} & z_{c b} & z_{d}
\end{array}\right], \\
z_{d}=r_{c}+r_{g}+j x_{d} ; \quad z_{s}=r_{g}+j x_{s} ; \quad z_{\mathrm{f}}=r_{\mathrm{g}}+j x_{\mathrm{f}} ; \\
z_{s f}=z_{s}-z_{f}=j x_{s}-j x_{f}=j x_{s f},
\end{gathered}
$$

where $r_{c}$ is active resistance of phase conductor, $r_{g}$ - ground resistance, $x_{d} ; x_{s}, x_{f}$ - self and mutual reactances.

In transposed lines mutual impedances (index ' $t$ ') are equal:

$$
z_{a b}=z_{a c}=z_{b a}=z_{b c}=z_{c a}=z_{c b}=z_{t},
$$

in Fortesque notation:

$$
Z_{t 012}=\left[\begin{array}{ccc}
Z_{0} & 0 & 0 \\
0 & Z_{1} & 0 \\
0 & 0 & Z_{2}
\end{array}\right],
$$


where:

$$
Z_{0}=z_{d}+2 z_{t} ; \quad Z_{1}=Z_{2}=Z_{d}-z_{t}
$$

Further on, quantities with index ' $s$ ' will pertain to Fig. 1a phase arrangement, with '. $m$ ' - to Fig. 1b arrangement if nothing is specified before.

Observing Fig. 1 and formula (3), we have:

$$
\boldsymbol{Z}_{a b c . s}=\left[\begin{array}{ccc}
z_{d} & z_{s} & z_{f} \\
z_{s} & z_{d} & z_{s} \\
z_{f} & z_{s} & z_{d}
\end{array}\right] ; \boldsymbol{Z}_{a b c . m}=\left[\begin{array}{ccc}
z_{d} & z_{s} & z_{s} \\
z_{s} & z_{d} & z_{f} \\
z_{s} & z_{f} & z_{d}
\end{array}\right]
$$

Inequality of mutual impedances creates mutual impedances $\left(Z_{01} ; Z_{02} ; Z_{10} ; Z_{12} ; Z_{20} ; Z_{21}\right)$ in Fortesque notation:

$$
Z_{012}=\left[\begin{array}{ccc}
Z_{0} & Z_{01} & Z_{02} \\
Z_{10} & Z_{1} & Z_{12} \\
Z_{20} & Z_{21} & Z_{2}
\end{array}\right]
$$

Relation between phase notation and Fortesque notation is:

$$
\boldsymbol{Z}_{012}=\boldsymbol{A} \boldsymbol{Z}_{a b c} \boldsymbol{A}^{-1}
$$

Basing on the expressions (3), (4), (7), and designations in Fig. 1, we receive expressions for Fortesque components of impedance in equation (5) for Fig. 1a:

$$
\begin{gathered}
Z_{0}=r_{c}+3 r_{g}+j\left(x_{d}+\frac{4}{3} x_{s}+\frac{2}{3} x_{f}\right) ; \\
Z_{01}=\frac{1}{2 \sqrt{3}} x_{s f}-j \frac{1}{6} x_{s f} \quad Z_{10}=-\frac{1}{2 \sqrt{3}} x_{s f}-j \frac{1}{6} x_{s f} ; \\
Z_{02}=-\frac{1}{2 \sqrt{3}} x_{s f}-j \frac{1}{6} x_{s f} Z_{20}=\frac{1}{2 \sqrt{3}} x_{s f}-j \frac{1}{6} x_{s f} ; \\
Z_{12}=-\frac{1}{\sqrt{3}} x_{s f}+j \frac{1}{3} x_{s f} \quad Z_{21}=\frac{1}{\sqrt{3}} x_{s f}+j \frac{1}{3} x_{s f} ; \\
Z_{1}=Z_{2}=r_{c}+j\left(x_{d}-\frac{2}{3} x_{s}-\frac{1}{3} x_{f}\right)
\end{gathered}
$$

and for Fig. 1b:

$$
\begin{gathered}
Z_{0}=r_{c}+3 r_{g}+j\left(x_{d}+\frac{4}{3} x_{s}+\frac{2}{3} x_{f}\right) \\
Z_{01}=Z_{10}=Z_{02}=Z_{20}=j \frac{1}{3} x_{s f} ; Z_{12}=Z_{21}=-j \frac{2}{3} x_{s f} \\
Z_{1}=Z_{2}=r_{c}+j\left(x_{d}-\frac{2}{3} x_{s}-\frac{1}{3} x_{f}\right)
\end{gathered}
$$

Self inductance $L_{d}$, mutual inductance $L_{m}$ and reactances [5] are:

$$
\begin{gathered}
L_{d}=0,4605\left(\lg \frac{D_{z}}{r}+0,05\right) 10^{-3}, \mathrm{H} / \mathrm{km} \\
L_{m}=0,4605 \lg \frac{D_{z}}{D_{p h-p h}} 10^{-3}, \mathrm{H} / \mathrm{km} \\
x=\varpi L, \Omega / \mathrm{km}
\end{gathered}
$$

where $r$ is phase conductor radius; $D_{z}$ is distance between phase conductor and return wire in the ground [6], $D_{z} \approx 930 \mathrm{~m}$; $D_{p h-p h}$, is distance between phase conductors; $\omega$ is circular frequency, $1 / \mathrm{s}$.

Since $D_{z}>D_{p h-p h}$, power line with phase conductors in the vertices of equilateral triangle can be considered as balanced with Fortesque impedances $Z_{0}$ and $Z_{1}=Z_{2}$ only.

Unbalanced line has additionally mutual impedances $Z_{01} \ldots$.

It should be noted that in flat arrangement of phases with lateral location of the phase ' $a$ ' mutual impedances change their value with the number order change in their indices.

In further considerations length of the line is taken $1 \mathrm{~km}$ in order to facilitate using the specific quantities of the line.

\section{IMPACT ON SingLE-PHASE-TO-EARTH FAULT}

For unbalanced lines, phase voltages can be expressed relying on (2):

$$
\boldsymbol{U}_{a b c}=\left[\begin{array}{l}
U_{a} \\
U_{b} \\
U_{c}
\end{array}\right]=\boldsymbol{A}^{-1} \boldsymbol{U}_{012}=\left[\begin{array}{ccc}
1 & 1 & 1 \\
1 & a^{2} & a \\
1 & a & a^{2}
\end{array}\right]\left[\begin{array}{c}
U_{0} \\
U_{1} \\
U_{2}
\end{array}\right],
$$

where

$$
\boldsymbol{U}_{012}=\left[\begin{array}{c}
U_{0} \\
U_{1} \\
U_{2}
\end{array}\right]=\left[\begin{array}{ccc}
Z_{0} & Z_{01} & Z_{02} \\
Z_{10} & Z_{1} & Z_{12} \\
Z_{20} & Z_{21} & Z_{2}
\end{array}\right]\left[\begin{array}{c}
I_{0} \\
I_{1} \\
I_{2}
\end{array}\right] .
$$

Then for phase 'a' observing (14) and (15) at single-phaseto-earth short circuit we can write:

$$
\begin{aligned}
& U_{a}=U_{p h}=U_{0}+U_{1}+U_{2}=Z_{0} I_{0}+Z_{01} I_{1}+Z_{02} I_{2}+ \\
& +Z_{10} I_{0}+Z_{1} I_{1}+Z_{12} I_{2}+Z_{20} I_{0}+Z_{21} I_{1}+Z_{2} I_{2}= \\
& =Z_{u 0} I_{0}+Z_{u 1} I_{1}+Z_{u 2} I_{2}
\end{aligned}
$$

where

$$
\begin{gathered}
Z_{u 0}=Z_{0}+Z_{10}+Z_{20} ; Z_{u 1}=Z_{01}+Z_{1}+Z_{2} ; \\
Z_{u 2}=Z_{02}+Z_{12}+Z_{2} .
\end{gathered}
$$

By single-phase-to-earth fault and by other types of faults (see further) their value depends on how the phase ' $a$ ' is located (see (11) and (12)).

For single-phase-to-earth fault [7], provision

$$
I_{0}=I_{1}=I_{2}=\frac{1}{3} I_{p h}
$$


holds, because condition

$$
I_{f B}=I_{f C}=U_{f A}=0
$$

is maintained.

Faulty phase current $I_{u p h}$ is:

$$
I_{u p h}=\frac{3 U_{p h}}{Z_{u \Sigma}},
$$

where total impedance $Z_{u \Sigma}$ is:

$$
Z_{u \Sigma}=Z_{u 0}+Z_{u 1}+Z_{u 2} .
$$

Observing (18), we can overwrite (16):

$$
U_{u p h}=Z_{u 1} I_{p h}\left[1+\frac{1}{3} \frac{Z_{u 0}-Z_{1}}{Z_{u 1}}+\frac{1}{3} \frac{Z_{u 2}-Z_{u 1}}{Z_{u 1}}\right]
$$

and further, introducing compensation factor $K_{u N}$,

$$
K_{u N}=\frac{Z_{u 0}+Z_{u 2}-2 Z_{u 1}}{3 Z_{u 1}}
$$

apparent impedance with metallic short circuit is:

$$
Z_{u a}=Z_{u 1}=\frac{U_{p h}}{I_{p h}\left(1+K_{u N}\right)}=\frac{Z_{u \Sigma}}{3\left(1+K_{u N}\right)} ;
$$

For transposed line well known formulas are:

$$
\begin{gathered}
Z_{t 0}=r_{c}+3 r_{g}+j\left(x_{d}+2 x_{t}\right) ; U_{p h}=\left(I_{1}+I_{2}\right) Z_{t 1}+I_{0} Z_{t 0} \\
Z_{t 1}=Z_{t 2}=r_{c}+j\left(x_{d}-x_{t}\right) ; K_{t N}=\frac{Z_{t 0}-Z_{t 1}}{3 Z_{t 1}} \\
Z_{t \Sigma}=Z_{t 0}+2 Z_{t 1} ; I_{t p h}=\frac{3 U_{p h}}{Z_{t \Sigma}} \\
Z_{t a}=\frac{U_{p h}}{I_{t p h}\left(1+K_{t N}\right)}=\frac{Z_{t \Sigma}}{3\left(1+K_{t N}\right)}
\end{gathered}
$$

Now we can compare $Z_{\text {ua.s }}, Z_{\text {ua.m }}, Z_{\text {ta }}$ and distance $L$ using the specific (per kilometer) values $Z_{s p}$ of impedances. Distance to fault place is determined using calculated $Z_{a p}\left(Z_{u a}\right.$ and $\left.Z_{t a}\right)$ and $Z_{s p} \quad\left(Z_{u s p}\right.$ and $\left.Z_{t . s p}\right)$, rather using their imaginable components (reactances). However at the influence assessment we can use impedances $Z_{a}$ and $Z_{s p}$ :

$$
l=\frac{Z_{a}}{Z_{s p}}
$$

Now we can compare $Z_{\text {иа.s }}, Z_{\text {иа.m }}, Z_{t a}$ and distance $L$ using the specific (per kilometer) values of just shown impedances. This will be done in section 5 .

\section{IMPACT ON BALANCED LOAD}

At the beginning, let us see what asymmetry appears with the three-phase short circuit at the end of the line. Phase currents are:

$$
\boldsymbol{I}_{a b c}=\boldsymbol{Z}_{a b c}{ }^{-1} \boldsymbol{U}_{a b c} ; \quad \boldsymbol{U}_{a b c}=\left[\begin{array}{c}
U_{a} \\
U_{b} \\
U_{c}
\end{array}\right]=\left[\begin{array}{c}
1 \\
a^{2} \\
a
\end{array}\right] .
$$

In Fortesque notation we have:

$$
\boldsymbol{I}_{012}=\left[\begin{array}{c}
I_{0} \\
I_{1} \\
I_{2}
\end{array}\right]=\boldsymbol{A} \boldsymbol{I}_{a b s} .
$$

Symmetrical components $I_{0}$ and $I_{2}$ show what asymmetry is created by untransposed power lines at three-phase short circuit.

To connect balanced load, means to acquire summary matrix $\boldsymbol{Z}_{L a b c \Sigma}$ by summarizing the branch impedance of matrix $\boldsymbol{Z}_{a b c}$ with diagonal load matrix $\boldsymbol{Z}_{L a b c}$ :

$$
\boldsymbol{Z}_{L a b c \Sigma}=\boldsymbol{Z}_{a b c}+\boldsymbol{Z}_{L a b c}
$$

where $\boldsymbol{Z}_{L a b c}$ consists of equal for all phases loads $Z_{L}$.

$$
Z_{L a b c}=\left[\begin{array}{ccc}
z_{L} & 0 & 0 \\
0 & z_{L} & 0 \\
0 & 0 & z_{L}
\end{array}\right]
$$

Load current observing (28) is:

$$
\boldsymbol{I}_{L a b c}=\boldsymbol{Z}_{L a b c \Sigma}{ }^{-1} \boldsymbol{U}_{a b c} .
$$

Asymmetry of voltage across the load can be determined as:

$$
\boldsymbol{U}_{L 012}=\boldsymbol{A} \boldsymbol{U}_{L . a b c} ; \boldsymbol{U}_{L a b c}=\boldsymbol{Z}_{L a b c} \boldsymbol{I}_{L . a b c} .
$$

\section{NUMERICAL RESULTS}

Calculations were made on the program Matlab.

A $110 \mathrm{kV}$ power line with flat arrangement of phases is taken for consideration. For phase conductor AC-300/39, specific resistance $r_{c}=0.098 \Omega / \mathrm{km}$; conductor radius $r=12 \mathrm{~mm}$.

Distance to imaginable wire in the ground $D_{z}=930 \mathrm{~m}$.

Distance $D_{p h-p h}$ between adjacent phases $D_{s}=4 \mathrm{~m}$, between lateral phases $D_{f}=8 \mathrm{~m}$.

Distance $D_{p f-p f}$ of transposed lines $D_{t}=1.26 \cdot D_{s}=5.04 \mathrm{~m}$.

Further, voltages are in V, currents in A, impedances - in $\Omega$.

On the basis of these data by (13) and (4) we have: $x_{d}=0.72269 ; x_{s}=0.3422 ; x_{f}=0.29866 ; x_{s f}=0.04354$; for transposed line $x_{p h-p h}=x_{t}=0.32766$.

Impact on single-phase-to-earth fault.

By (17) observing (11) $Z_{u 0 . s}=0.248+j 1.3636$;

$Z_{u 1 . s}=0.1357+j 0.4023 ; Z_{u 2 . s}=0.0603+j 0.4023$.

By (21) $Z_{u \Sigma . s}=0.444+j 2.1681$; 
By $(23) K_{u N . s}=0.7244+j 0.2138$

Apparent impedance by (24) is $Z_{\text {uа.s }}=0.1357+j 0.4023 \mathrm{i}$;

By (27) distance to the fault is $l_{s}=1$;

By (17) observing (12) $Z_{u 0 . m}=0.248+j 1.4071$; $Z_{u 1 . m}=0.098+j 0.3805 ; Z_{u 2 . m}=0.098+j 0.3805$.

By (21) $Z_{u \Sigma . m}=0.444+j 2.1681$;

By $(23) K_{u N . m}=0.8752+j 0.094$;

Apparent impedance by (24) is $Z_{\text {ua.m }}=0.098+j 0.3805$;

By (27) distance to the fault is $l_{m}=1$;

By $(25) Z_{t 0}=0.248+j 1.378 ; \quad Z_{t 1}=0.098+j 0.395 ;$

$Z_{t \Sigma}=0.444+j 2.1681 ; \quad K_{t N}=0.8109+j 0.0746$;

By (26) apparent impedance $Z_{t a}=1$;

By (27) distance to the fault is $l_{t}=1$.

The results show that for single-phase metallic short circuit in untransposed power lines, the error do not appear if faulty phase (lateral or middle) and its specific parameters $\left(Z_{u}\right)$ are chosen by digital protection correctly.

Calculation of three phase short circuit current.

By (4) $z_{d}=0.148+\mathrm{j} 0.7227 ; z_{s}=0.05+j 0.3422 ; z_{f}=0.05+j 0.2987$.

For Fig. 1a, observing (8), (28), (4), we have:

$$
\boldsymbol{I}_{a b c . s}=\boldsymbol{Z}_{a b c . s}{ }^{-1}\left[\begin{array}{c}
1 \\
a^{2} \\
a
\end{array}\right]=\left[\begin{array}{c}
0.7215-2.2471 i \\
-2.5240+0.6809 i \\
1.8842+1.5594 i
\end{array}\right] \text {. }
$$

Symmetrical components $\boldsymbol{I}_{012 . s}$ by (29), their absolute value $\left|\boldsymbol{I}_{012 . s}\right|$ and current unbalance factors in percents (relative to first component) are:

$\boldsymbol{I}_{012 s}=\left[\begin{array}{c}0.0273-0.0023 i \\ 0.6007-2.3950 i \\ 0.0935+0.1501 i\end{array}\right] ;\left|\boldsymbol{I}_{012 s}\right|=\left[\begin{array}{l}0.0274 \\ 2.4692 \\ 0.1769\end{array}\right] ;\left[\begin{array}{l}1.11 \\ 7.16\end{array}\right]$.

The same, calculated for Fig. 1b, is:

$\boldsymbol{I}_{012 m}=\left[\begin{array}{c}-0.0116+0.0248 i \\ 0.6007-2.3950 i \\ 0.0833+0.1562 i\end{array}\right] ;\left|\boldsymbol{I}_{012 m}\right|=\left[\begin{array}{l}0.0274 \\ 2.4692 \\ 0.1770\end{array}\right] ;\left[\begin{array}{l}1.11 \\ 7.19\end{array}\right]$.

Symmetrical component proportion can be considered the same for special phase conductor lateral and middle arrangement which corresponds to physical sense.

Calculation of loaded power line.

The resistance of balanced active load is assumed tenfold of $Z_{1}=Z_{2}$ active component $\operatorname{Re}\left(Z_{1}\right)=R_{1}$. By $(11)$ $Z_{1}=0.098+j 0.395$, hence load resistance $r_{L}=10 \cdot 0.098=0.98$; by (31)

$$
\boldsymbol{R}_{\text {Labc }}=\left[\begin{array}{ccc}
0.98 & 0 & 0 \\
0 & 0.98 & \\
0 & 0 & 0.98
\end{array}\right]
$$

by (30)

$$
\begin{aligned}
& \boldsymbol{Z}_{\text {Labc.s }}=\boldsymbol{Z}_{a b c . s}+\boldsymbol{R}_{\text {Labc }}= \\
& {\left[\begin{array}{ccc}
1.128+j 07227 & 0.05+j 0.3422 & 0.05+j 0.2987 \\
0.05+j 0.3422 & 1.128+j 0.7227 & 0.05+j 03422 \\
0.05+j 0.2987 & 0.05+j 0.3422 & 1.128+j 0.7227
\end{array}\right] .}
\end{aligned}
$$

Branch current observing (28);
$\boldsymbol{I}_{\text {Labc.s }}=\left[\begin{array}{c}0.7967-0.2884 i \\ -0.6594-0.5723 i \\ -0.1347+0.8813 i\end{array}\right]$.

Symmetrical components $\boldsymbol{I}_{L 012 . s}$ by (29), their absolute value $\left|\boldsymbol{I}_{L 012 . s}\right|$ and current unbalance factor are:

$$
\boldsymbol{I}_{L 012 s}=\left[\begin{array}{c}
0.0008+0.0068 i \\
0.8176-0.2991 i \\
-0.0217+0.0039 i
\end{array}\right] ;\left|\boldsymbol{I}_{L 012 S}\right|=\left[\begin{array}{c}
0.0069 \\
0.8706 \\
0.0221
\end{array}\right] ;\left[\begin{array}{l}
0.79 \\
2.54
\end{array}\right] \text {. }
$$

If active load is twice of the previous value, the zero and second sequences are $0.95 \%$ and $4.11 \%$. Thus, with increasing load, energy quality deteriorates. It could be concluded even without doubling the load because with short circuit at the end of the line these figures were $1.1 \%$ and $7.16 \%$.

Inductive load of the same value generates $\mathrm{I}_{0}=0.62 \%$, $\mathrm{I}_{2}=2.1 \%$, capacitive load gives $\mathrm{I}_{0}=2.94 \%, \mathrm{I}_{2}=4.95 \%$.

Voltages at the end of the line have the same proportions since they are equal to current vector matrix multiplied by diagonal load resistance matrix with equal phase values.

Calculation of symmetrical components for Fig. $1 \mathrm{~b}$ gives the same result.

\section{IMPACT ON OTHER TYPES OF SHORT CIRCUIT}

The effect on three-phase fault protection can be seen in previous section calculating three-phase fault current. The zero sequence is roughly $1 \%$ but not so easy it is with the second sequence which is more than $7 \%$. However this drawback can be eliminated by filtering or by per phase settings.

Phase-to-phase fault.

Preconditions [7]

$$
I_{A}=0 ; I_{B}=-I_{C} ; U_{B}-U_{C}=0
$$

hold. Since zero sequence is absent, hence

$$
I_{1}=-I_{2} ; E-U_{1}=-U_{2} .
$$

Observing (15) and (35):

$$
\begin{aligned}
& U_{1}=Z_{1} I_{1}+Z_{12} I_{2}=\left(Z_{1}-Z_{12}\right) I_{1}=Z_{u 1} I_{1} ; \\
& U_{2}=Z_{21} I_{1}+Z_{2} I_{2}=\left(Z_{2}-Z_{21}\right) I_{2}=Z_{u 2} I_{2} .
\end{aligned}
$$

since

$$
Z_{u 1}=Z_{1}-Z_{12} ; Z_{u 2}=Z_{2}-Z_{21}
$$

and positive sequence of current is:

$$
I_{1}=\frac{E}{Z_{u 1}+Z_{u 2}} .
$$

With specific values of $Z_{u 1}$ and $Z_{u 2}$ known, further procedures is the same as at transposed lines.

Phase-to-phase-to-earth fault.

Preconditions [7] hold

$$
I_{A}=U_{B}=U_{c}=0 .
$$


Since

$$
I_{1}=-\left(I_{0}+I_{2}\right) ; U_{1}=U_{0}=U_{2} ; E_{A}-U_{1}=U_{0} .
$$

Observing (15) and (40) we have:

$$
\begin{gathered}
Z_{0} I_{0}+Z_{01} I_{1}+Z_{02} I_{2}=Z_{20} I_{0}+Z_{21} I_{1}+Z_{2} I_{2} \\
Z_{0} I_{0}+Z_{01}\left(-I_{0}-I_{2}\right)+Z_{02} I_{2}=Z_{20} I_{0}+Z_{21}\left(-I_{0}-I_{2}\right)+Z_{2} I_{2} \\
\left(Z_{0}-Z_{01}-Z_{20}+Z_{21}\right) I_{0}=\left(Z_{2}-Z_{21}-Z_{02}+Z_{01}\right) I_{2} \\
Z_{u 0}=Z_{0}-Z_{01}-Z_{20}+Z_{21} \\
Z_{u 2}=Z_{2}-Z_{21}-Z_{02}+Z_{01}
\end{gathered}
$$

Based on (40), we can write:

$$
I_{0}=\frac{Z_{u 2}}{Z_{u 0}+Z_{u 2}}\left(-I_{1}\right) ; I_{2}=\frac{Z_{u 0}}{Z_{u 0}+Z_{u 2}}\left(-I_{1}\right) \text {. }
$$

Observing (15) and (42), we have:

$$
U_{1}=\left(-\frac{Z_{10} Z_{u 2}}{Z_{u 0}+Z_{u 2}}+Z_{1}-\frac{Z_{12} Z_{u 0}}{Z_{u 0}+Z_{u 2}}\right) I_{1}
$$

and untransposed line first sequence impedance equals:

$$
Z_{u 1}=-\frac{Z_{10} Z_{u 2}}{Z_{u 0}+Z_{u 2}}+Z_{1}-\frac{Z_{12} Z_{u 0}}{Z_{u 0}+Z_{u 2}} .
$$

Further steps are the same as at transposed power lines.

Hence relay protection of untransposed lines will not have errors if specific impedances $Z_{u}$ will be written in the protection according to the type of fault and special phase ' $a$ ' displacement (lateral or middle).

The measures to diminish the effect of unbalance in digital relay protection is easier for realisation when the line is not transposed because the settings of the detected faulty phase can be changed regardless of the distance to the fault while in transposed line they should be changed depending on what side of transposition place fault occurs.

Load is always affected positively by transposition.

\section{INFLUENCE OF STRAY CAPACITANCE}

Verification is needed to determine whether to pay attention to stray capacitances (Fig. 2) examining untransposed lines.

Specific [6] capacitive coefficients between phase conductor and earth $\alpha_{a a}$, between adjacent phase conductors $\alpha_{a b}$ and between lateral conductors $\alpha_{a c}$ are:

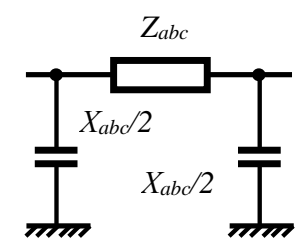

Fig. 2. Stray capacitances of the power line displayed as lump quantities. where $\mathrm{r}$ is conductor radius, $h$ is wire height above ground, $D_{a b}$ is distance between adjacent wires, $D_{a c}$ is distance between lateral wires, $H_{a b}$ is distance between phase 'a' wire and the reflection of phase 'b' wire, $D_{a b}$ - the same for 'a' and 'c' [6].

It is convenient here to use capacitance (capacitive impedance) $\chi$ which is equal:

$$
\chi=\alpha / \omega .
$$

Then we have capacitive impedance matrix (for Fig. 1a):

$$
\boldsymbol{X}_{a b c}=\left[\begin{array}{lll}
\chi_{a a} & \chi_{a b} & \chi_{a c} \\
\chi_{b a} & \chi_{b b} & \chi_{b c} \\
\chi_{c a} & \chi_{c b} & \chi_{c c}
\end{array}\right]=\left[\begin{array}{lll}
\chi_{d} & \chi_{s} & \chi_{f} \\
\chi_{s} & \chi_{d} & \chi_{s} \\
\chi_{f} & \chi_{s} & \chi_{d}
\end{array}\right]
$$

Internal impedance $Z_{a b c . i}$ of the line is the impedance of connected in parallel $Z_{a b c}$ and $X_{a b c} / 2$ :

$$
Z_{a b c i}=\frac{Z_{a b c}\left(\mathrm{X}_{a b c} / 2\right)}{Z_{a b c}+\mathrm{X}_{a b c} / 2}
$$

The task is to determine how $Z_{a b c . i}$ differs from $Z_{a b c}$.

For power line considered in section 5 , we have additionally the following dimensions: $h=6 \mathrm{~m} ; H_{a b}=12.65 \mathrm{~m} ; H_{a c}=14.42 \mathrm{~m}$; $D_{a b}=D_{s} ; D_{a c}=D_{f}($ see section 5$)$.

By (45) and (46) we have: $\chi_{a a}=\chi_{d}=-j 395540 \Omega$; $\chi_{a b}=\chi_{s}=-j 65924 \Omega ; \chi_{a c}=-j 33739 \Omega$.

Verification by (48) showed that $Z_{a b c . i}$ begins to differ from $\boldsymbol{Z}_{a b c}$ only when $\boldsymbol{X}_{a b c}$ is diminished a hundred times.

Corona conductance does not differ much from admitted even for $330 \mathrm{kV}$ lines, since it does not have influence as well. Even if the impact would be, it can not be investigated as there is no data on self and mutual conductance.

\section{ALTERNATIVE FOR TRANSPOSITION}

If unbalanced line with symmetrical load generates asymmetrical phase currents, then asymmetrical load at the end of unbalanced line can generate symmetrical phase currents. Summary matrix (30) with unbalanced load $z_{a}, z_{b}, z_{c}$ looks:

$$
\boldsymbol{Z}_{L a b c \Sigma}=\left[\begin{array}{ccc}
z_{L a} & z_{s} & z_{f} \\
z_{s} & z_{L b} & z_{s} \\
z_{f} & z_{s} & z_{L c}
\end{array}\right]=\left[\begin{array}{ccc}
z_{d}+z_{a} & z_{s} & z_{f} \\
z_{s} & z_{d}+z_{b} & z_{s} \\
z_{f} & z_{s} & z_{d}+z_{c}
\end{array}\right]
$$

where $z_{a}, z_{b}, z_{c}$ are loads of phases a, b, c at line end.

According to [8] inverse matrix $\boldsymbol{Z}_{L a b c \Sigma^{-1}}$ is:

$$
\boldsymbol{Z}_{L a b c \Sigma}{ }^{-1}=\frac{1}{\operatorname{det}\left(\boldsymbol{Z}_{L a b c \Sigma}\right)}\left[\begin{array}{ccc}
\alpha_{a} & \beta & \gamma \\
\beta & \alpha_{b} & \delta \\
\gamma & \delta & \alpha_{c}
\end{array}\right]
$$

where 
Load current multiplied by $\operatorname{det}\left(\boldsymbol{Z}_{L a b c \Sigma}\right)$, observing (28), is:

$$
\boldsymbol{I}_{L a b c}{ }^{\prime}=\boldsymbol{I}_{L a b c} \operatorname{det}\left(\boldsymbol{Z}_{L a b c \Sigma}\right)=\left[\begin{array}{c}
\alpha_{a}+a^{2} \beta+a \gamma \\
\beta+a^{2} \alpha_{b}+a \delta \\
\gamma+a^{2} \delta+a \alpha_{c}
\end{array}\right] .
$$

Voltage at power line end $\boldsymbol{U}_{\text {end }}$ ' multiplied by $\operatorname{det}\left(\boldsymbol{Z}_{L a b c \Sigma}\right)$ is:

$$
\boldsymbol{U}_{\text {end }}{ }^{\prime}=\left[\begin{array}{ccc}
Z_{a} & 0 & 0 \\
0 & Z_{b} & 0 \\
0 & 0 & Z_{c}
\end{array}\right] \boldsymbol{I}_{L a b c}{ }^{\prime}=\left[\begin{array}{c}
\left(\alpha_{a}+a^{2} \beta+a \gamma\right) Z_{a} \\
\left(\beta+a^{2} \alpha_{b}+a \delta\right) Z_{b} \\
\left(\gamma+a^{2} \delta+a \alpha_{c}\right) Z_{c}
\end{array}\right] .
$$

Voltage at the end of untransposed power line will be balanced when between phase voltages is such coherence:

$$
U_{\text {end.a }}=a U_{\text {end. } b}=a^{2} U_{\text {end.c }},
$$

i.e.

$$
\left(\alpha_{a}+a^{2} \beta+a \gamma\right) Z_{a}=\left(a \beta+\alpha_{b}+a^{2} \delta\right) Z_{b}=\left(a^{2} \gamma+a \delta+\alpha_{c}\right) Z_{c}
$$

To obtain target, two conditions can be set up observing (53):

$$
U_{\text {end.a }}=a U_{\text {end. } b} ; U_{\text {end.a }}=a^{2} U_{\text {end.c }} .
$$

Since only two conditions exist, in one phase only the load must be chosen, wherein it is considerable that the loads in other two phases be mostly convenient for implementation in compliance with the requirement of all three phases summary load.

\section{CONCLUSIONS}

1. Unbalanced power lines without $O G W$ have three basic Fortesque impedances $Z_{0}$; and $Z_{1}=Z_{2}$ and six more mutual impedances $Z_{01}, Z_{10}, Z_{02}, Z_{20}, Z_{12}, Z_{21}$ whose value depends on phase ' $a$ ' lateral or middle location in flat arrangement of phase conductors. It is the result of different branch mutual reactances.

2. Magnitude of negative and zero sequence at the end of untransposed power line increases with load increasing. $110 \mathrm{kV}$ line with $X / R=4$, loaded by active load with resistance tenfold of phase conductor active resistance generates $0.79 \%$ of zero sequence and $2.54 \%$ of negative sequence; same value inductive load gives $0.62 \%$ and $2.1 \%$ respectively; capacitive load $-2.94 \%$ and $4.95 \%$.

3. Stray capacity and corona losses are too low to influence asymmetry.

4. Under asymmetric short circuits, impact on relay protection depends on location of special phase: whether it is located laterally or in the middle of flat arrangement of phase conductors.
5. Digital relay protection can easier cope with the line phase unbalance effect when it is untransposed.

6. Balanced voltage at the end of untransposed power line can be obtained connecting unbalanced load.

\section{REFERENCES}

[1] A. von Jouanne and B. Banerjee, "Assessment of Voltage Unbalance", Power Delivery, IEEE Transactions on, vol. 16, no. 4, pp. 782-790, October, 2001.http://dx.doi.org/10.1109/61.956770

[2] P. Paranavithana, S. Perera, D. Sutanto, "Impact of Untransposed $66 \mathrm{kV}$ Sub-transmission lines on Voltage Unbalance," 2007. [Online]. Available: http://ro.uow.edu.au/cgi/viewcontent.cgi?article=8290\&context=engpap ers. Accessed on: Oct. 2, 2014.

[3] P. Giridhar Kini, R. C. Bansal and R. S. Aithal, "Impact of voltage unbalance on the performance of three-phase induction motor," The South Pacific Journal of Natural Science, vol. 24, no. 1, pp. 45-50, 2006. [Online]. Available: http://www.publish.csiro.au/?act=view_file\&file_id=SP06007.pdf. http://dx.doi.org/10.1071/SP06007

[4] F. Calero. "Mutual Impedance in Parallel Lines - Protective relaying and Fault Location Considerations," pp. 1-15, TP6283-01, 2007. [Online]. Available: $w w w . s e l i n c . c o m / W o r k A r e a / D o w n l o a d A s s e t . a s p x ? i d=3488$.

[5] J. Schlabbach, K-H. Rofalski. Power System Engineering, $1^{\text {th }} \mathrm{ed}$. Weinheim: WILEY-VCH Verlag GnbH \& Co. KGaA, 2008, ch. 13, pp. 223-233

[6] B. Li, F. Guo, X. Li, Z. Bo. "Circulating Unbalanced Current of EHV/UHV Untransposed Double-Circuit Lines and Their Influence on Pilot Protection," Power Delivery, IEEE Transactions on, vol. 29, no. 2, pp. 825-833, Dec., 2013. [Online]. Available: https://www.deepdyve.com/ lp/institute-of-electrical-and-electronics-engineers/circulating-unbalancedcurrents-of-ehv-uhv-untransposed-double-circuit-7sFn8UnS52. Accessed on: March 20, 2014. http://dx.doi.org/10.1109/TPWRD.2013.2286092

[7] A. Wang, Q. Chen, Z. Zhou. „Effects of untransposed UHV transmission line on fault analysis of power systems," in transactions of Tianjin University, vol. 14, no. 3, pp. 231-234, June 2008. http://dx.doi.org/10.1007/s12209-008-0042-6

[8] A. M. Gashimov, A. R. Kabayeva, A. Nayir. Transmission line transposition. Electrical and Electronics Engineering, 2009. ELECO 2009. International Conference on, pp. 364-367, Nov., 2009. [Online]. Available: www.emo.org.tr/ekler/97a39505016683c_ek.pdf.

[9] Y. Hase. Handbook of Power System Engineering. Chichester, West Sussex, England: John Wiley \& Sons Ltd, 2008, pp.1-44.

[10] A. Vanags. Elektriskie tīkli un sistēmas. Rīga: RTU, 2005, lpp. 120-127, 133-138 (in Latvian).

[11] A. Ulyanov. Elektromagnitniye perehodniye processy v elektricheskich sistemach. Moskva: Energiya, 1970, pp. 316-322 (in Russian).

[12] Invertible matrix - Wikipedia, the free encyclopedia. [Online]. Available: en.wikipedia.org/wiki/invertible_matrix.

Josifs Survilo. Born in Kraslava, Latvia in 1936 March 26. PhD, defended in 1980 at Urals Polytechnic Institute in the field "Power systems and the control of them." Dr. sc. ing. obtained in Riga Technical University, Latvia in 2001. After graduating from Riga Polytechnic Institute in 1960 for 40 years have been working as an engineer at Riga works "Energoautomatika." Since 2000 is working in Riga Technical University as a researcher.

Previous publications: Survilo J. Impact of distributed generation on current and impedance protections. RTU $54^{\text {th }}$ International Scientific Conference (D:) Proceedings, Latvia: 14-16 October, 2013. Survilo J. Account of losses in electricity sales. RTU $54^{\text {th }}$ International Scientific Conference (D:) Proceedings, Latvia: 14-16 October, 2013. Survilo J. Reciprocal load-generator power losses in a grid. Latvian Journal of Physics and Technical Sciences, No. 6, pp. 48-53; 2013.

Address: Kronvalda 1, LV-1010, Riga.

Phone:+371 67089939,

E-mail: jahzeps@eef.rtu.lv 